\title{
Atypical enhanced computed tomography signs of pancreatic cancer and its differential diagnosis from autoimmune pancreatitis
}

\author{
Yong Zhao" ${ }^{1 \#}$, Fei Li ${ }^{2 \#}$, Ning $\mathrm{An}^{3}$, Zehua Peng ${ }^{4}$ \\ ${ }^{1}$ Department of Emergency, Sichuan Academy of Medical Sciences \& Sichuan Provincial People's Hospital (East Hospital), Chengdu, China; \\ ${ }^{2}$ Department of Hepatobiliary Surgery, Sichuan Academy of Medical Sciences \& Sichuan Provincial People's Hospital (East Hospital), Chengdu, \\ China; ${ }^{3}$ Department of Hepatobiliary Surgery, Sichuan Academy of Medical Sciences \& Sichuan Provincial People's Hospital, Chengdu, China; \\ ${ }^{4}$ Department of Radiology, Sichuan Academy of Medical Sciences \& Sichuan Provincial People's Hospital, Chengdu, China \\ Contributions: (I) Conception and design: Y Zhao, F Li; (II) Administrative support: N An, Z Peng; (III) Provision of study materials or patients: N \\ An, Z Peng; (IV) Collection and assembly of data: Y Zhao, F Li; (V) Data analysis and interpretation: Y Zhao, F Li; (VI) Manuscript writing: All \\ authors; (VII) Final approval of manuscript: All authors. \\ "These authors contributed equally to this work. \\ Correspondence to: Zehua Peng. Department of Radiology, No.32 West Section 2, First Ring Road, Chengdu 610072, China. Email: pengzh0915@126.com; \\ Ning An. Department of Hepatobiliary Surgery, No.32 West Section 2, First Ring Road, Chengdu 610072, China. Email: anningsyy321@sina.com.
}

Background: To analyze the atypical enhanced computed tomography (CT) signs of pancreatic cancer (PC) and compare them with those of autoimmune pancreatitis (AIP) to explore the differential diagnosis value of CT.

Methods: The clinical data of 36 AIP (AIP group) and 38 PC patients (PC group), who were admitted to our hospital from January 2013 to June 2020 and confirmed by surgical biopsy or hormone therapy, were retrospectively analyzed. Participants in both groups were examined by CT, the imaging signs of the 2 groups were analyzed, and the results of CT examination were compared.

Results: In the PC group, the density of the lesions on the CT scan was mostly reduced, the pancreas was not swollen, and the kidneys were not involved. The bile duct wall was thickened with a sausage-like appearance, enveloped edges were rare, blood vessels were invaded, lymph nodes were enlarged, and the pancreatic duct was truncated. The findings of the AIP group were the opposite. The difference in the proportion of participants with the above-mentioned CT features between the 2 groups was statistically significant $(\mathrm{P}<0.05)$. The shape of the lesions in the AIP group was mainly elongated, of uneven density, and the density of enhanced scanning was medium to high. The predominant shape of the lesions in PC participants was spherical, and the density was uniform. The enhanced scan was mainly low-density. The difference in shape and density between the 2 groups was also statistically significant $(\mathrm{P}<0.05)$. The CT values of the plain scan, intravenous phase, and delayed phase in the AIP group were significantly higher than those in the PC group $(\mathrm{P}<0.05)$.

Conclusions: The imaging signs of AIP and PC overlap. Examination with CT is of great value in the differential diagnosis between AIP and PC. Familiarity with and mastery of the CT signs of AIP and PC can help to improve the accuracy of clinical diagnosis and provide a reliable basis for patients' follow-up treatment.

Keywords: Autoimmune pancreatitis (AIP); pancreatic cancer (PC); differential diagnosis; imaging signs

Submitted Oct 16, 2020. Accepted for publication Dec 28, 2020.

doi: $10.21037 / \mathrm{gs}-20-821$

View this article at: http://dx.doi.org/10.21037/gs-20-821 


\section{Introduction}

Autoimmune pancreatitis (AIP) is a special type of chronic pancreatitis mediated by autoimmunity and characterized by pancreatic enlargement and irregular narrowing of the pancreatic duct $(1,2)$. Lymphoid plasma cell infiltration with pancreatic tissue fibrosis is the main pathological feature of AIP. At the same time, pancreatic tissue fibrosis can lead to impaired pancreatic function. Therefore, early and accurate diagnosis can allow patients to receive effective drug treatment and avoid unnecessary surgery.

Pancreatic duct stenosis, chronic pancreatitis, and localized pancreatic duct stenosis are the main types of AIP. Among them, the imaging and clinical features of pancreatic cancer (PC) overlap with localized pancreatic duct stenosis, leading to difficulty in clinical differential diagnosis, and a high rate of misdiagnosis $(3,4)$. Furthermore, there are vast differences in the treatment of PC and AIP. Misdiagnosis will thus delay the patient's condition and threaten their life. Imaging examination is a common auxiliary method for clinical differential diagnosis of pancreatic diseases.

AIP is an uncommon form of chronic pancreatitis. Whilst being corticosteroid responsive, AIP often masquerades radiologically as pancreatic neoplasia. The mean accuracies for diagnosing AIP and PC are $68 \%$ and $83 \%$, respectively. The most common findings for AIP are common bile duct (CBD) stricture, bile duct wall hyperenhancement, and diffuse parenchymal enlargement. The most common findings for PC are focal mass and pancreatic ductal dilatation. Misdiagnosis of $\mathrm{PC}$ in patients with AIP is due to focal mass, pancreatic duct dilatation, and pancreatic atrophy, whereas misdiagnosis of AIP in patients with PC is due to absence of atrophy, presence of diffuse enlargement, and peripancreatic halo (5). Multidetectorrow computed tomography (MDCT) and magnetic resonance (MR) imaging are currently the most frequently performed imaging modalities for the study of pancreatic disease. In all the cases in which AIP is strongly suspected but the diagnosis is uncertain, MDCT and MR are useful imaging modalities to suggest the correct diagnosis. Repeated MDCT and/or MR examinations after short-term treatment (2-3 weeks) with high-dose steroids can identify a significant reduction in the size of pancreatic parenchyma, and the normalisation of the calibre of the MPD and bile ducts. The results of this short-term follow-up are obviously present in AIP and absent in the case of pancreatic adenocarcinoma (6). Previous research have exhibited that the five methods including multidetector computed tomography (MDCT) , contrast-enhanced high mechanical index EUS (CEHMI-EUS), EUS, B-Mode EUS and contrast-enhanced low mechanical index EUS (CELMIEUS) were performed to examine the pancreatic carcinoma, CEHMI-EUS is considered a standard procedure when pancreatic carcinoma is suspected in a patient (7). The enhanced duct sign was more frequently observed in patients with AIP than in patients with PC or chronic pancreatitis (8). Among them, computed tomography (CT) examination is widely used in clinical practice due to its low price, fast scanning speed, and wide range of applications (9). Therefore, this study analyzed PC atypical enhanced CT signs through retrospective investigation and compared them with AIP to explore the differential diagnosis value of CT, to provide a reliable basis for clinical diagnosis and treatment. We present the following article in accordance with the MDAR checklist (available at http://dx.doi. org/10.21037/gs-20-821).

\section{Methods}

\section{General information}

We conducted a retrospective analysis of the clinical data of 36 AIP and 38 PC patients admitted to our hospital between January 2013 and June 2020 and confirmed by surgical biopsy or hormone therapy. All experiments were carried out in accordance with the Declaration of Helsinki (as revised in 2013). All patients agreed to participate in this study and signed an informed consent form. This study was approved by the Sichuan Academy of Medical Sciences \& Sichuan Provincial People's Hospital (No. H20130527). The inclusion criteria for this study were as follows: (I) AIP patients met the relevant diagnostic criteria in the Japanese Acute Pancreatitis Treatment Guidelines (10); (II) eligible for CT examination; (III) complete imaging and pathological data; (IV) without other pancreatic diseases; (V) no history of alcohol abuse or gallstones. The exclusion criteria were as follows: (I) patients with liver and kidney dysfunction; (II) patients with intestinal obstruction, cholecystitis, and other diseases; (III) patients with autoimmune systemic diseases; (IV) patients who had undergone/were undergoing preoperative chemotherapy and radiotherapy. With the above criteria, the eligible participants were 22 males and 14 females with AIP, aged $35-78$ years old, with an average age of $55.62 \pm 5.54$ years. Their clinical symptoms included abdominal pain, diarrhea, hypoglycemia, jaundice, and other symptoms. There were 
23 males and 15 females of the eligible PC patients, aged $38-77$ years, with an average age of $55.82 \pm 5.26$ years. Their clinical symptoms included abdominal pain, diarrhea, weight loss, palpable mass, and jaundice.

\section{Study method}

We examined and excluded all metals on the participant that may have affected the scan. The participant took the supine position the supine anatomical position. A GE Light speed 64-slice spiral CT scanner (GE Medical Systems, Milwaukee, WI, USA) was used for imaging. Participants were requested to fast for $>8 \mathrm{~h}$ before the operation and drink $1,000 \mathrm{~mL}$ of water $30 \mathrm{~min}$ before the examination to ensure the stomach and duodenum were full. The scanning started from the top of the diaphragm and descended to all parts of the pancreas. The scanning parameters included tube voltage $120 \mathrm{KV}$, tube current $150 \mathrm{~mA}$, scanning layer thickness $5 \mathrm{~mm}$, pitch 1.0, and reconstruction interval $5 \mathrm{~mm}$. An extra $3 \mathrm{~s}$ were allowed before the scan to prepare for the patient's breath-holding. A routine plain scan was first performed, and then a high-pressure syringe was used to inject $80 \mathrm{~mL}$ of iohexol via the elbow vein for a dynamic three-phase enhanced scan, with an injection rate of $3.0 \mathrm{~mL} / \mathrm{s}$. The delayed scan time for the arterial phase was $25-30 \mathrm{~s}$, the delayed scan time for the venous phase was $65 \mathrm{~s}$, and the delayed phase was $120 \mathrm{~s}$. After the scan was completed, the multi-slices helical CT (MSCT) postprocessing workstation was used to reconstruct the coronal and sagittal images of the participant's axial scan.

\section{Observation indicators}

The CT images were read by 2 senior and experienced radiologists in the diagnostic team, focusing on the following CT signs: (I) the shape of the lesion. The pancreas is divided into 3 parts, the focal type is defined when the lesion only affects 1 local-part; the diffuse type is defined when the lesion affects $\geq 2$ consecutive parts and with a sausage-like appearance and fibrous capsule; (II) the shape of the pancreas. Whether there were signs of lymph node enlargement (lymph node shorter diameter $>1.0 \mathrm{~cm}$ ), vascular involvement (the lesion was wrapped around by a blood vessel, or the blood vessel was twisted); (III) whether there was a thickening of the bile duct wall (bile duct wall thickening $>0.3 \mathrm{~cm}$ ), bile duct dilation (pancreatic part of the bile duct or concentric stenosis, upper pancreatic head lumen $>1.0 \mathrm{~cm}$ ) and pancreatic duct dilation (pancreatic duct lumen $>0.3 \mathrm{~cm}$ ), among other signs.

The calcification, density, and kidney involvement of the 2 groups of participants were observed, and changes in morphology, density, and CT values of the 2 groups were compared.

\section{Statistical analysis}

The data in this study were analyzed with the statistical software SPSS version 22.0 (SPSS Inc., IBM Corp., Chicago, IL, USA). The measurement data were described as the mean \pm standard deviation (SD), and the $t$-test was used for comparison. Counting data were expressed by composition ratio, and the chi-squared $\left(\chi^{2}\right)$ test was used for comparison. The difference was considered statistically significant when $\mathrm{P}<0.05$.

\section{Results}

\section{General information of the 2 groups of patients}

Comparison of the general information of the 2 groups of patients showed no difference in general data such as gender, age, course of the disease, and history between the 2 groups, and they were comparable $(\mathrm{P}>0.05)$, as shown in Table 1.

\section{Comparison of CT scan results between the 2 groups of patients}

Comparison of CT scan results between the 2 groups revealed that in the PC group, the density of the lesions on the CT scan was mostly decreased, the pancreas was not swollen, and the kidneys were not involved. The bile duct wall was thickened, of sausage-like appearance, and envelope-like edges were rare. The blood vessels were invaded, lymph nodes were enlarged, and the pancreatic duct was truncated. The AIP group was just the opposite (Figure 1A,B). The difference in the proportion of the abovementioned CT features between the 2 groups was statistically significant $(\mathrm{P}<0.05)$, see Table 2 .

\section{Comparison of CT imaging characteristics between the 2 groups}

Comparison of CT imaging features between the 2 groups showed that the shape of the lesions in PC patients was mainly 
Table 1 General information of the 2 groups of patients $[(\bar{x} \pm s), \mathrm{n}(\%)]$

\begin{tabular}{|c|c|c|c|c|c|}
\hline \multirow{2}{*}{ Group } & \multirow{2}{*}{ Male/female } & \multirow{2}{*}{ Average age (years) } & \multirow{2}{*}{$\begin{array}{l}\text { Disease course } \\
\text { (years) }\end{array}$} & \multicolumn{2}{|c|}{ History } \\
\hline & & & & Hypertension & Diabetes \\
\hline AIP group $(n=36)$ & $22 / 14$ & $55.62 \pm 5.54$ & $0.87 \pm 0.35$ & 5 & 3 \\
\hline PC group (n=38) & $23 / 15$ & $55.82 \pm 5.26$ & $0.92 \pm 0.31$ & 6 & 4 \\
\hline$t / \chi^{2}$ & 0.003 & 0.159 & 0.651 & \multicolumn{2}{|c|}{0.168} \\
\hline
\end{tabular}

AIP, autoimmune pancreatitis; PC, pancreatic cancer.
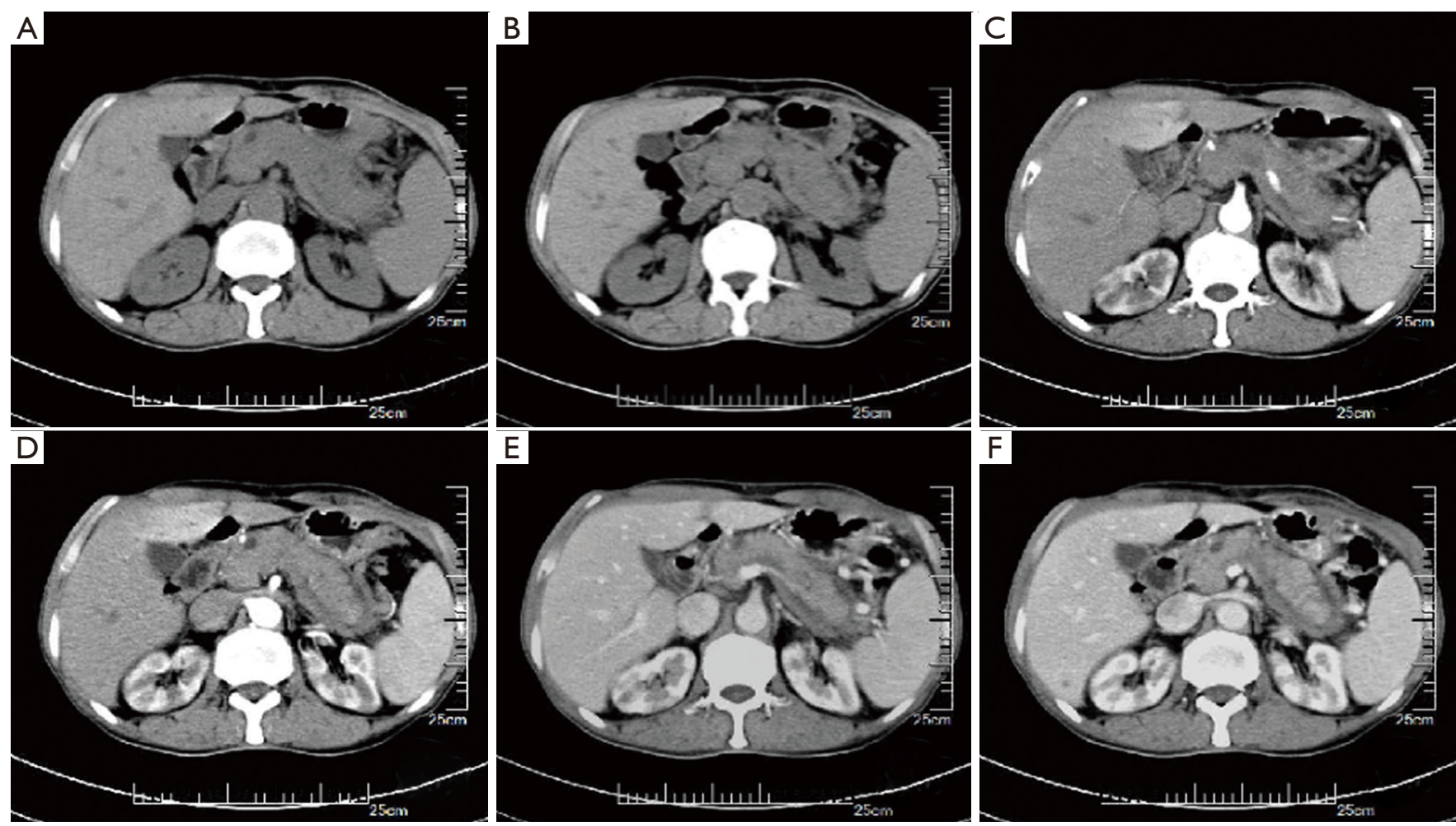

Figure 1 The results of CT scan. (A,B) The AIP CT scan shows pancreatic enlargement, the body tail is obvious, boundary is blurred, parenchymal density is slightly reduced, and the body tail is surrounded by a slightly low-density shadow. (C,D) The AIP enhanced scan for the late arterial phase, the pancreas is slightly enhanced and the density is slightly uneven. (E,F) Enhanced scan for the portal vein stage, the pancreas shows uneven enhancement, which is significantly lower than that of the liver, and thin strips of low-density shadows are seen inside. CT, computed tomography; AIP, autoimmune pancreatitis.

spherical, of uniform density, and the enhanced scan was mainly low-density, whereas the shape of the lesions in the AIP group was mainly long, of uneven density, and the density of the enhanced scan was moderately high (Figure 1C,D,E,F). The difference in morphology and density between the 2 groups was statistically significant $(\mathrm{P}<0.05)$, see Table 3 .

\section{Comparison of CT values between the 2 groups}

Comparison of CT values between the 2 groups revealed that the CT values of the plain scan, arterial phase, venous phase, and delayed phase in the AIP group were significantly higher than those in the PC group. The CT values of the plain scan, venous phase, and delayed phase 
Table 2 Comparison of CT scan results between the 2 groups of patients [n (\%)]

\begin{tabular}{lcccccccccc}
\hline Group & $\begin{array}{c}\text { Reduced } \\
\text { lesion } \\
\text { density }\end{array}$ & $\begin{array}{c}\text { Swollen } \\
\text { pancreas }\end{array}$ & $\begin{array}{c}\text { Capsular } \\
\text { edge }\end{array}$ & $\begin{array}{c}\text { Thickening } \\
\text { bile duct } \\
\text { wall }\end{array}$ & $\begin{array}{c}\text { Pancreatic } \\
\text { duct } \\
\text { truncation }\end{array}$ & $\begin{array}{c}\text { Vascular } \\
\text { invasion }\end{array}$ & $\begin{array}{c}\text { Enlarged } \\
\text { lymph } \\
\text { nodes }\end{array}$ & $\begin{array}{c}\text { Sausage- } \\
\text { like } \\
\text { appearance }\end{array}$ & $\begin{array}{c}\text { Pancreatic } \\
\text { duct } \\
\text { dilation }\end{array}$ & $\begin{array}{c}\text { Kidney } \\
\text { involvement }\end{array}$ \\
\hline $\begin{array}{l}\text { AIP group } \\
(\mathrm{n}=36)\end{array}$ & $7(19.44)$ & $27(75.00)$ & $23(63.89)$ & $23(63.89)$ & $4(11.11)$ & $0(0.00)$ & $7(19.44)$ & $30(83.33)$ & $32(88.89)$ & $12(33.33)$ \\
$\begin{array}{l}\text { PC group } \\
(\mathrm{n}=38)\end{array}$ & $38(100.00)$ & $0(0.00)$ & $2(5.26)$ & $3(7.89)$ & $34(89.47)$ & $25(65.79)$ & $17(44.74)$ & $3(7.89)$ & $33(86.84)$ & $0(0.00)$ \\
$\chi^{2}$ & 50.338 & 44.872 & 28.403 & 25.432 & 45.441 & 35.768 & 5.397 & 42.580 & 0.072 & 15.118 \\
$P$ & 0.001 & 0.001 & 0.001 & 0.001 & 0.001 & 0.001 & 0.020 & 0.001 & 0.788 & 0.001 \\
\hline
\end{tabular}

AIP, autoimmune pancreatitis; PC, pancreatic cancer.

Table 3 Comparison of CT imaging characteristics between the 2 groups [n (\%)]

\begin{tabular}{|c|c|c|c|c|c|c|c|c|}
\hline Group & \multicolumn{2}{|c|}{ Shape } & \multicolumn{3}{|c|}{ Density uniformity } & \multicolumn{3}{|c|}{ Enhanced scan density } \\
\hline $\begin{array}{l}\text { AIP group } \\
(n=36)\end{array}$ & $2(5.56)$ & $34(94.44)$ & $0(0.00)$ & $36(100.00)$ & $0(0.00)$ & $14(38.89)$ & $21(58.33)$ & $1(2.78)$ \\
\hline $\begin{array}{l}\text { PC group } \\
(n=38)\end{array}$ & 36 (94.74) & $2(5.26)$ & $35(92.11)$ & $3(7.89)$ & $0(0.00)$ & $36(94.74)$ & $2(5.26)$ & $0(0.00)$ \\
\hline$P$ & \multicolumn{2}{|c|}{0.001} & \multicolumn{3}{|c|}{0.001} & \multicolumn{3}{|c|}{0.001} \\
\hline
\end{tabular}

CT, computed tomography; AIP, autoimmune pancreatitis; PC, pancreatic cancer.

were significantly different between the 2 groups $(\mathrm{P}<0.05)$, see Table 4.

\section{Discussion}

As one of the benign pancreatic lesions, AIP is easily misdiagnosed as PC in clinical practice. The etiology and pathogenesis of the disease have not yet been fully elucidated. It is generally believed that the occurrence of AIP is related to pancreatic involvement when the patient's immune system is over-reactive $(11,12)$. Onset is more common in elderly, predominantly male patients. This study also consisted of predominantly male participants, which mirrored the real-life situation.

The common pathological changes in AIP patients are pancreatic enlargement and pancreatic duct stenosis. After the onset, there are no specific clinical symptoms, however, it can manifest as abdominal pain and obstructive jaundice (13). Additionally, AIP often leads to extrapancreatic involvement, which intensifies the suffering of patients and further complicates clinical treatment.
The main categories of AIP are diffuse and localized, among which the incidence of diffuse AIP is the highest and the most common site of AIP is the head of the pancreas. An important imaging feature is focal low-density swelling, which overlaps to some degree with the imaging signs of PC, thus often misleading the clinical differential diagnosis $(14,15)$. The incidence of $\mathrm{PC}$ in pancreatic malignancies is as high as $90 \%$, and it has also gradually increased in recent years. Due to the concealed position of the pancreas and rapid pathological progress, the treatment and prognosis of this disease are poor (16). In the clinical treatment of PC, surgical treatment is often used, supplemented by chemotherapy and radiotherapy when necessary, while the AIP mainly uses steroid therapy, and the specific conservative treatment plan is often adjusted according to the follow-up observation results. Usually, surgical treatment is not recommended. Therefore, there are obvious differences between the 2 diseases regarding treatment (17). If AIP and PC can be diagnosed accurately at an early stage, it will assist the formulation of targeted treatment plans and improvement of the efficacy and quality 
Table 4 Comparison of CT values between the 2 groups $\left(x_{ \pm} \mathrm{SD}\right)$

\begin{tabular}{lcccc}
\hline Group & Plain scan & Arterial phase & Venous phase & Delayed phase \\
\hline AIP group $(n=36)$ & $40.53 \pm 7.65$ & $67.28 \pm 21.17$ & $81.18 \pm 18.10$ & $82.61 \pm 18.42$ \\
PC group $(n=38)$ & $34.24 \pm 6.43$ & $62.03 \pm 16.15$ & $67.46 \pm 12.68$ & $65.85 \pm 11.26$ \\
$t$ & 3.836 & 1.203 & 3.793 & 4.751 \\
$P$ value & 0.001 & 0.233 & 0.001 & 0.001 \\
\hline
\end{tabular}

CT, computed tomography; AIP, autoimmune pancreatitis; PC, pancreatic cancer; SD, standard deviation.

of medical treatment.

At present, the accurate differential diagnosis of AIP and PC before surgery has become a hot topic for clinicians and scholars. The value of CT examination in the diagnosis and staging of PC has been clinically recognized, and it is regarded as the best imaging method for PC examination $(18,19)$. In recent years, CT has been used increasingly in the differential diagnosis of AIP and PC, and the CT characteristics of the 2 diseases are significantly different. The CT signs of AIP are mainly pancreatic swelling. At the same time, the density of the lesions is reduced, bile duct wall is thickened, blood vessels are invaded, and the lymph nodes are enlarged.

The results of this study showed that there were significant differences in pancreatic swelling, kidney involvement, bile duct wall thickening, sausage-like appearance, envelope-like edges, vascular invasion, and enlarged lymph nodes between the 2 groups, but there was no significant difference in pancreatic duct dilation between the 2 groups. Muraki et al. (20) reported that the edge of the capsule can be observed in about $16-80 \%$ of AIP patients. Heo et al. (21) and other studies have suggested that the changes of the biliary system have a high reference value in the differential diagnosis of AIP. The findings of this study showed that the AIP group mainly displayed thickening of the bile duct wall, while the CT signs of the bile duct in the PC group were mainly pancreatic duct truncation. Therefore, AIP can be exonerated when pancreatic duct truncation occurs on CT, but if there is bile duct wall thickening accompanied by high density lesions, AIP can be highly suspected (22). The CT value of the AIP group during the plain scan was significantly higher than that of the PC group, and after the enhanced scan, the AIP group showed progressive and uniform enhancement of the disease, while the PC group had no obvious enhancement, which was consistent with the report of Vujasinovic et al. (23). In the present study, we also found that there were significant differences in the CT values of AIP and CP during the plain scan, venous phase scan, and delayed phase scan, which suggested that the CT value of lesions is of great significance in the differential diagnosis.

Currently, AIP and PC can be diagnosed clinically based on patient symptoms, signs, and typical imaging signs. However, it has been found from previous literature and our current study that there are still some cases of atypical PC that are difficult to distinguish from the imaging signs of AIP. Therefore, the appearance of the thickening of the bile duct wall, pancreatic duct truncation, and other elements (24) can be integrated with other clinical data to differentiate between AIP and PC.

In summary, the imaging signs of AIP and PC overlap. Examination using CT is of great value in the differential diagnosis of these two diseases. Familiarity with and masteryof the CT signs of AIP and PC can help improve the accuracy of clinical diagnosis and provide a reliable basis for the optimizing patient follow-up treatment.

\section{Acknowledgments}

Funding: None.

\section{Footnote}

Reporting Checklist: The authors have completed the MDAR checklist. Available at http://dx.doi.org/10.21037/gs-20-821

Data Sharing Statement: Available at http://dx.doi. org/10.21037/gs-20-821

Conflicts of Interest: All authors have completed the ICMJE uniform disclosure form (available at http://dx.doi. org/10.21037/gs-20-821). The authors have no conflicts of interest to declare.

Ethical Statement: The authors are accountable for all aspects of the work in ensuring that questions related 
to the accuracy or integrity of any part of the work are appropriately investigated and resolved. All experiments were carried out in accordance with the Declaration of Helsinki (as revised in 2013). All patients agreed to participate in this study and signed an informed consent form. This study was approved by the Sichuan Academy of Medical Sciences \& Sichuan Provincial People's Hospital (No. H20130527).

Open Access Statement: This is an Open Access article distributed in accordance with the Creative Commons Attribution-NonCommercial-NoDerivs 4.0 International License (CC BY-NC-ND 4.0), which permits the noncommercial replication and distribution of the article with the strict proviso that no changes or edits are made and the original work is properly cited (including links to both the formal publication through the relevant DOI and the license). See: https://creativecommons.org/licenses/by-nc-nd/4.0/.

\section{References}

1. Zhang J, Jia G, Zuo C, et al. 18F-FDG PET/CT helps differentiate autoimmune pancreatitis from pancreatic cancer. BMC Cancer 2017;17:695.

2. Zheng L, Xing H, Li F, et al. Focal Autoimmune Pancreatitis Mimicking Pancreatic Cancer on FDG PET/ CT Imaging. Clin Nucl Med 2018;43:57-9.

3. Hsu WL, Chang SM, Wu PY, et al. Localized autoimmune pancreatitis mimicking pancreatic cancer: Case report and literature review. J Int Med Res 2018;46:1657-65.

4. Wang M, Huang S, Pei R, et al. Endoscopic ultrasonography guided transgastric trans-portal system fine needle aspiration for diagnosing pancreatic head and uncinate process malignancy. Ann Transl Med 2019;7:719.

5. Zaheer A, Singh VK, Akshintala VS, et al. Differentiating autoimmune pancreatitis from pancreatic adenocarcinoma using dual-phase computed tomography. J Comput Assist Tomogr 2014;38:146-52.

6. Graziani R, Mautone S, Ambrosetti MC, et al. Autoimmune pancreatitis: multidetector-row computed tomography (MDCT) and magnetic resonance (MR) findings in the Italian experience. Radiol Med 2014;119:558-71.

7. Harmsen FR, Domagk D, Dietrich CF, et al. Discriminating chronic pancreatitis from pancreatic cancer: Contrast-enhanced EUS and multidetector computed tomography in direct comparison. Endosc Ultrasound 2018;7:395-403.
8. Kawai Y, Suzuki K, Itoh S, et al. Autoimmune pancreatitis: assessment of the enhanced duct sign on multiphase contrast-enhanced computed tomography. Eur J Radiol 2012;81:3055-60.

9. Miyoshi H, Kano M, Kobayashi S, et al. A Case of Diffuse Pancreatic Cancer Mimicking Autoimmune Pancreatitis. Intern Med 2019;58:1646-8.

10. Lee S, Kim JH, Kim SY, et al. Comparison of diagnostic performance between CT and MRI in differentiating nondiffuse-type autoimmune pancreatitis from pancreatic ductal adenocarcinoma. Eur Radiol 2018;28:5267-74.

11. Ito T, Kawa S, Matsumoto A, et al. Risk Factors for Pancreatic Stone Formation in Type 1 Autoimmune Pancreatitis: A Long-term Japanese Multicenter Analysis of 624 Patients. Pancreas 2019;48:49-54.

12. Seleznik GM, Reding T, Peter L, et al. Development of autoimmune pancreatitis is independent of CDKN1A/p21mediated pancreatic inflammation. Gut 2018;67:1663-73.

13. Kim JH, Byun JH, Kim MH, et al. Pancreatic Duct in Autoimmune Pancreatitis: Intraindividual Comparison of Magnetic Resonance Pancreatography at $1.5 \mathrm{~T}$ and $3.0 \mathrm{~T}$. Pancreas 2017;46:921-6.

14. Liu M, Hao M. Unique properties of IgG4 antibody and its clinical application in autoimmune pancreatitis. Scand J Gastroenterol 2018;53:1121-31.

15. Dite P, Novotny I, Dvorackova J, et al. Pancreatic Solid Focal Lesions: Differential Diagnosis between Autoimmune Pancreatitis and Pancreatic Cancer. Dig Dis 2019;37:416-21.

16. Dickerson LD, Farooq A, Bano F, et al. Differentiation of Autoimmune Pancreatitis from Pancreatic Cancer Remains Challenging. World J Surg 2019;43:1604-11.

17. Daniel DL, Ahmed F, Farhat B, et al. Differentiation of autoimmune pancreatitis from pancreatic cancer remains challenging. Pancreatology 2018;18:S117.

18. Saraireh HA, Bilal M, Parupudi S, et al. Autoimmune Pancreatitis Mimicking Pancreatic Cancer: It Can Happen. Am J Gastroenterol 2017;112:S711.

19. Sugimoto M, Takagi T, Suzuki R, et al. Endoscopic Ultrasonography-Guided Fine Needle Aspiration Can Be Used to Rule Out Malignancy in Autoimmune Pancreatitis Patients. J Ultrasound Med 2017;36:2237-44.

20. Muraki T, Kim GE, Reid MD, et al. Paraduodenal Pancreatitis: Imaging and Pathologic Correlation of 47 Cases Elucidates Distinct Subtypes and the Factors Involved in its Etiopathogenesis. Am J Surg Pathol 2017;41:1347-63.

21. Heo WG, Kim TH, Kim YJ, et al. Autoimmune 
Pancreatitis Complicated With Pancreatic Ascites, Pancreatic Ductal Leakage, and Multiple Pseudocyst. Pancreas 2017;46:e10-1.

22. Zhang Y, Cheng C, Liu Z, et al. Radiomics analysis for the differentiation of autoimmune pancreatitis and pancreatic ductal adenocarcinoma in 18 F-FDG PET/CT. Med Phys 2019;46:4520-30.

23. Vujasinovic M, Valente R, Maier P, et al. Diagnosis,

Cite this article as: Zhao Y, Li F, An N, Peng Z. Atypical enhanced computed tomography signs of pancreatic cancer and its differential diagnosis from autoimmune pancreatitis. Gland Surg 2021;10(1):347-354. doi: 10.21037/gs-20-821 treatment and long-term outcome of autoimmune pancreatitis in Sweden. Pancreatology 2018;18:900-4.

24. Schneider A, Hirth M, Münch M, et al. Risk of Cancer in Patients with Autoimmune Pancreatitis: A Single-Center Experience from Germany. Digestion 2017;95:172-80.

(English Language Editor: J. Jones) 